\title{
EXISTENCE AND UNIQUENESS FOR A BOUSSINESQ SYSTEM WITH A DISORDERED FORCING *
}

\author{
JOSÉ R. QUINTERO ${ }^{\dagger}$ AND JUAN C. MUÑOZ
}

\begin{abstract}
We study the existence, uniqueness, regularity and continuous dependence on initial data of solutions for the Cauchy problem associated with the coupled system of Boussinesq type equations forced by highly oscillatory smooth coefficients. The model considered describes two-way propagation of long water waves with small amplitude on the surface of a one-dimensional channel with rough bottom (disordered topography). The dependent variables in this model are the wave elevation $\eta$ and the potential velocity $u$ measured at the fixed depth $Z_{0}=\sqrt{2 / 3}$.
\end{abstract}

1. Introduction. In this paper we are concerned with global existence, uniqueness, regularity and continuous dependence on initial data of solutions to the Boussinesq type system

$$
\left\{\begin{array}{c}
M(\xi) \eta_{t}+\partial_{\xi}\left[\left(1+\frac{\alpha \eta}{M(\xi)}\right) u\right]-\frac{\beta}{6} \partial_{\xi}^{2}\left(M(\xi) \eta_{t}\right)=0,(\xi, t) \in \mathbb{R} \times[0, \infty) \\
u_{t}+\eta_{\xi}+\frac{\alpha}{2} \partial_{\xi}\left[\left(\frac{u}{M(\xi)}\right)^{2}\right]-\frac{\beta}{6} \partial_{\xi}^{2}\left(u_{t}\right)=0, \quad(\xi, t) \in \mathbb{R} \times[0, \infty) \\
\eta(\xi, 0)=f(\xi), \quad u(\xi, 0)=g(\xi), \quad \xi \in \mathbb{R},
\end{array}\right.
$$

where the forcing coefficient $M(\xi)$ is a $C^{\infty}$ bounded function with the property $\inf _{\mathbb{R}} M(\xi)>0$, and $\alpha$ and $\beta$ are positive constants.

The system above governs the two-way propagation of small amplitude water waves on the surface of a wide class of bottom profiles, including highly oscillatory topographies (i.e. when the scale of variation of the bottom irregularities is small compared to the typical wavelength observed on the water surface) [11]. It extends the regime of application of other Boussinesq formulations for variable depth, such as those by Peregrine [14] and Nwogu [13], which are valid provided that the mild slope restriction holds (c.f. Mei [8], pag. 59).

Formally, the system in (1.1) is a weakly dispersive $(\beta<<1)$, weakly nonlinear $(\alpha<<1)$ approximation of the potential theory equations for an irrotational, incompressible and inviscid fluid ([17] [11]). The function $u(\xi, t)$ represents the potential velocity measured at a fixed station located at the depth $Z_{0}=\sqrt{2 / 3}$. The function $\eta(\xi, t)$ denotes the wave elevation measured with respect to the undisturbed free surface. The function $M(\xi)$ in model (1.1) is topography dependent and it can be characterized as a convolution between the function describing the coast depth and a $C^{\infty}$ kernel. In case of constant depth, the coefficient $M(\xi)$ is identically one and the Boussinesq system (1.1) reduces to a system considered by Bona and Chen ([3]).

The computational complexity of system (1.1) is comparable to that of the terrainfollowing Boussinesq system [12]. However, we point out that the symmetric dispersive terms in the motion equations in system (1.1) make easier to develop efficient and unconditionally stable Boussinesq solvers for system (1.1). For instance, Bona and Chen ([3]) derived a fourth order accurate and unconditionally stable scheme

\footnotetext{
*Received October 24, 2003; accepted for publication February 26, 2004.

$\dagger$ Universidad del Valle, A.A. 25360, Cali, Colombia (quinthen@univalle.edu.co).

$\ddagger$ Universidad del Valle, A.A. 25360, Cali, Colombia (jcarlmz@yahoo.com).
} 
to compute numerical solutions of system (1.1) in a channel of constant depth (i.e., when $M(\xi) \equiv 1)$ and arbitrary finite length. This is an important feature to be taken into account in water wave simulations over large time intervals. We notice that this scheme could be adapted for computing solutions of system (1.1) when the bottom is rough. In contrast, the numerical solver employed in [10] for approximating solutions of the terrain-following Boussinesq system ([12]) is conditionally stable. This imposes a restriction on the size of the temporal and spatial grid points adopted in the finite difference scheme in order to guarantee that the error produced by discretization does not grow as long as time increases.

We also want to emphasize that the curvilinear coordinate system introduced in the derivation of system (1.1) could remove the stiffness phenomenon (promoted by a possible disordered topography) in numerical simulations for system (1.1). Based on these facts, we consider the system (1.1) particularly interesting in computer simulations of weakly dispersive, weakly nonlinear waves propagating over a disordered bottom. Details, properties and numerical experiments for the Boussinesq system (1.1) will appear elsewhere [11].

Several authors have investigated the existence of solutions of Boussinesq type equations for water waves propagating over shallow channels of constant depth, in which coefficients in the motion equations considered are constant. Bona and Smith showed the global existence, uniqueness and regularity of solutions and continuous dependence of solutions on initial conditions for a Boussinesq model modeling twoway wave propagation in a channel of constant depth ([2]). Also, Bona and Chen studied the existence of solitary wave solutions of a family of Boussinesq models, by using the concentration-compactness principle ([4]). Amick [1] and Schonbek [15] analyzed the global existence, uniqueness and regularity of solutions for a Boussinesq system of equations derived by Whitham ([17], page 466). On the other hand, large time behavior of solutions for a generalization of the Boussinesq system of equations (with dissipation) in dimension $n \geq 2$, is explored by Rajopadhye et. al. ([16]). However, theory on the existence and uniqueness of solutions for a Boussinesq system forced by a highly oscillatory bottom, like the system (1.1) considered in the paper, has not been addressed yet, to our knowledge. In a nutshell, the goal in this paper is to start the study of the properties of the solutions for models, which include some complicated physical phenomenon as dispersive waves in highly variable media.

Before we go on, we want to point out some features of the Boussinesq system (1.1), which are clever in our analysis. First, as mentioned above, the function $M(\xi)$ in the coefficients of the system (1.1) is infinitely differentiable even though the function describing the depth is discontinuous or non-differentiable. Moreover, the coefficient $M(\xi)$ is time independent, which makes the system (1.1) invariant under translations in the time variable $t$. Second, the presence of the regularizing dispersive terms $\partial_{\xi}^{2}\left(M(\xi) \eta_{t}\right)$ and $\partial_{\xi}^{2}\left(u_{t}\right)$, in both the mass and momentum conservation equations in (1.1), is crucial in the discussion of the local existence result. Third, we find a positive energy type functional (depending on the coefficient $M(\xi)$ ) which is conserved in time on solutions of a system equivalent to equations (1.1). The existence of this nonlinear functional together with the fact that the variable coefficient $M(\xi)$ is time independent allows us to derive bounds for the wave elevation and the fluid velocity valid for all time and extend the local solutions in time. This is a desirable property for a model approximating water waves and a theoretical advantage of (1.1) with respect to other models for arbitrary depths, such as that derived recently by Nachbin, for which is unclear the existence of a conserved positive quantity ([12]). The technique used to 
derive our results was inspired by the work of Bona and Smith in [2].

The motivation for the present study relies on the variety of recent applications of Boussinesq type systems considering rough bottom. It is important to note that these shallow water models are not only of interest in ocean exploration but also in meteorology where the waves propagate in the atmosphere. Some of these models have been explored by using alternative techniques such as asymptotic approximation, stochastic analysis and numerical simulation. For instance, in [9] is studied the O'Doherty-Anstey and the stabilization phenomena for dispersive waves propagating along a channel with a highly variable depth, by using the terrain-following Boussinesq formulation presented in [12]. In [10] is explored numerically the refocusing property of solitary waves (i.e. waveform inversion), promoted by a one-dimensional disordered media. Fouque, Garnier and Nachbin derived a stochastic theory for the refocusing and pulse stabilization of weakly dispersive waves in a one-dimensional random media $([6])$.

This paper is organized as follows. In Section 2, we present an outline of the derivation of the Boussinesq system (1.1). As done by Hamilton in [7], a convenient conformal curvilinear coordinates system is introduced to transform the physical channel onto a strip. As a result, the function describing the physical depth is substituted by the smooth coefficient $M(\xi)$ in the resulting motion equations (1.1). In Section 4 , we introduce the main spaces necessary to study the existence and regularity of solutions of system (1.1). Furthermore, we rewrite it as a pair of integral equations, convenient for the analysis in later sections. In Section 5 we discuss the uniqueness of a solution pair $(\eta, u)$ of system (1.1). In Section 6 , we prove the local existence of solutions by applying the contraction mapping principle to the integral operator associated with system (1.1). The regularity of the solutions is considered in Section 7. In Section 8 we extend the local solutions in time by using a nonlinear functional which conserves in time on solutions for a system equivalent to equations (1.1). Finally, in Section 9 we analyze the continuous dependence of a solution of system (1.1) on the initial data $f(\xi)$ and $g(\xi)$. The conclusions are given in Section 10.

2. Governing equations. We start by presenting the potential theory formulation for Euler's equations (in dimensionless variables) with a free surface and an impermeable bottom topography [17]:

$$
\beta \phi_{x x}+\phi_{y y}=0 \text { for }-H(x / \gamma)<y<\alpha \eta(x, t), \quad-\infty<x<\infty,
$$

with the nonlinear free surface conditions

$$
\begin{aligned}
\eta_{t}+\alpha \phi_{x} \eta_{x}-\frac{1}{\beta} \phi_{y} & =0, \\
\eta+\phi_{t}+\frac{\alpha}{2}\left(\phi_{x}^{2}+\frac{1}{\beta} \phi_{y}^{2}\right) & =0
\end{aligned}
$$

at $y=\alpha \eta(x, t)$. Here $\phi(x, y, t)$ denotes the potential velocity and $\eta(x, t)$ the wave elevation measured with respect to the undisturbed free surface $y=0$. The dimensionless parameters $\alpha$ and $\beta$ measure the strength of nonlinear and dispersive effects. The parameter $\gamma$ measures the ratio inhomogeneities/wavelength. The Neumann condition at the impermeable bottom is

$$
\phi_{y}+\frac{\beta}{\gamma} H^{\prime}(x / \gamma) \phi_{x}=0
$$


The bottom topography is described by $y=-H(x / \gamma)$ where

$$
H(x / \gamma)=\left\{\begin{array}{l}
1+n(x / \gamma), \quad \text { when } 0<x<L \\
1, \quad \text { when } x \leq 0 \text { or } x \geq L .
\end{array}\right.
$$

The bottom profile is described by the (possibly rapidly varying) function $-n(x / \gamma)$. The topography is rapidly varying when $\gamma \ll 1$. The scale $L$ represents the total length of the irregular section of the coast. The undisturbed depth is given by $y=-1$ and the topography can be of large amplitude provided that $|n|<1$. The fluctuations $n$ are not assumed to be small, nor continuous, nor slowly varying.

Consider a symmetric flow domain by reflecting the original one about the undisturbed free surface (c.f. Figure 2.1). This domain is denoted by $\Omega_{z}$ where $z=x+i \sqrt{\beta} y$ and can be considered as the conformal image of the strip $\Omega_{w}$ where $w=\xi+i \tilde{\zeta}$ with $|\tilde{\zeta}| \leq \sqrt{\beta}$. Then $z=x(\xi, \tilde{\zeta})+i \sqrt{\beta} y(\xi, \tilde{\zeta})=x(\xi, \tilde{\zeta})+i \tilde{y}(\xi, \tilde{\zeta})$ with $x$ and $\tilde{y}$ a pair of harmonic functions on $\Omega_{w}$. Following the strategy suggested by Hamilton in [7], the potential theory equations can be cast in the orthogonal curvilinear coordinates $(\xi, \tilde{\zeta})$ as [12]:

$$
\beta \phi_{\xi \xi}+\phi_{\zeta \zeta}=0, \quad \text { at } \quad 0<\zeta<1+\alpha N(\xi, t)
$$

with free surface conditions

$$
\begin{aligned}
|J| N_{t}+\alpha \phi_{\xi} N_{\xi}-\frac{1}{\beta} \phi_{\zeta} & =0, \quad \text { at } \quad \zeta=1+\alpha N(\xi, t) \\
\eta+\phi_{t}+\frac{\alpha}{2|J|}\left(\phi_{\xi}^{2}+\frac{1}{\beta} \phi_{\zeta}^{2}\right) & =0, \quad \text { at } \quad \zeta=1+\alpha N(\xi, t) \\
\phi_{\zeta} & =0, \quad \text { at } \quad \zeta=0,
\end{aligned}
$$

where we defined $\zeta=\tilde{\zeta} / \sqrt{\beta}+1$. This change of variables lets the origin of the curvilinear coordinate system at the bottom. The Jacobian for the $(\xi, \tilde{\zeta}) \rightarrow(x, \tilde{y})$ coordinate transformation is represented by $|J|$, and $\zeta=1+\alpha N(\xi, t)$ corresponds to the position of the free surface in the curvilinear coordinate system.

By performing an asymptotic simplification in the equations (2.5)-(2.8) within weakly nonlinear $(\alpha<<1)$, weakly dispersive $(\beta<<1)$ regime, we find that the free surface conditions (2.6)-(2.7) can be approximated to order $O(\alpha), O(\beta)$ by the equations

$$
\begin{array}{r}
\eta+f_{t}-\frac{\beta}{2} f_{\xi \xi t}+\frac{\alpha}{2 M^{2}(\xi)} f_{\xi}^{2}=O\left(\alpha \beta, \beta^{2}\right), \\
M(\xi) \eta_{t}+\left[\left(1+\frac{\alpha}{M(\xi)} \eta\right) f_{\xi}\right]_{\xi}-\frac{\beta}{6} f_{\xi \xi \xi \xi}=O\left(\alpha^{2}, \alpha \beta, \beta^{2}\right),
\end{array}
$$

where $M(\xi) \equiv \tilde{y}_{\tilde{\zeta}}(\xi, 0)=1+m(\xi)$ with,

$$
m(\xi ; \sqrt{\beta}, \gamma) \equiv \frac{\pi}{4 \sqrt{\beta}} \int_{-\infty}^{\infty} \frac{n\left(x\left(\xi_{0},-\sqrt{\beta}\right) / \gamma\right)}{\cosh ^{2} \frac{\pi}{2 \sqrt{\beta}}\left(\xi_{0}-\xi\right)} d \xi_{0}=(K *(n \circ x))(\xi) .
$$

Furthermore, $f(\xi, t)=\phi(\xi, 0, t)$ represents the potential velocity at the bottom. We point out that equations (2.9) and (2.10) correspond to those derived in [12] (bottom 


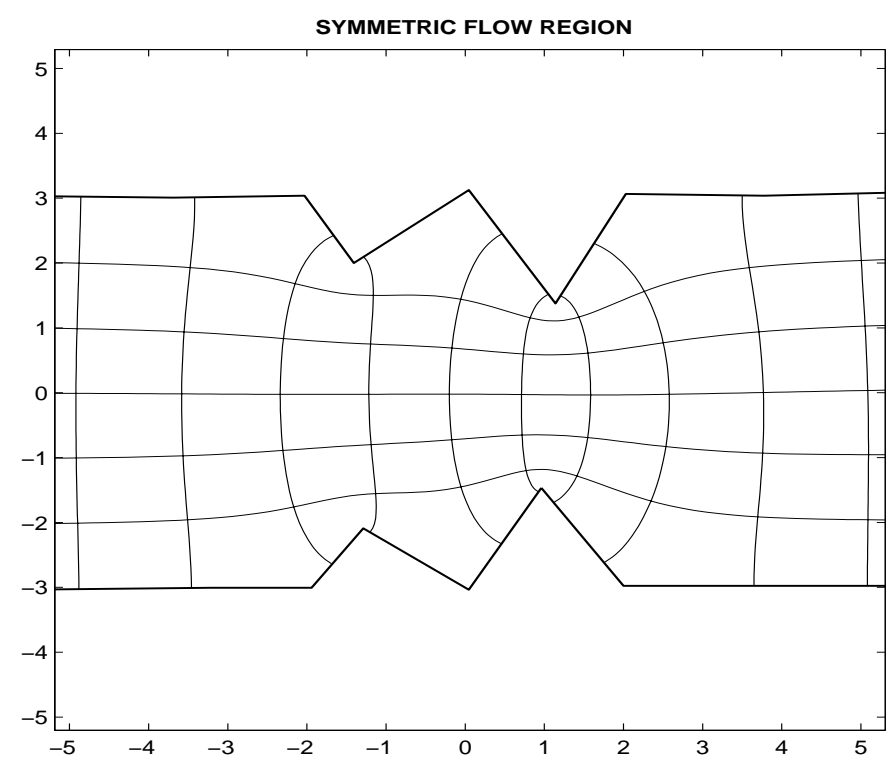

FIG. 2.1. The symmetric domain in the complex $z$-plane, where $z=x(\xi, \tilde{\zeta})+i \tilde{y}(\xi, \tilde{\zeta})$. The lower half $(x \in[-5,5], \quad y \in[-3,0])$ is the physical channel with $y=\tilde{\zeta}=0$ indicating the undisturbed free surface. Superimposed in this complex z-plane domain are the (curvilinear) coordinate level curves from the $w$-plane system $\xi \tilde{\zeta}$. The polygonal line at the bottom of the figure is a schematic representation of the topography (where $\tilde{\zeta}= \pm \sqrt{\beta}$ ). This figure was generated using SC-Toolbox [5].

of page 915 and top of page 916). In the derivation of equations (2.9)-(2.10) we used the relationship

$$
|J|(\xi, t)=M(\xi)^{2}+O\left(\alpha^{2}\right),
$$

which means that, at leading order, the Jacobian of the conformal coordinate transformation is time independent. Note that the coefficient $M(\xi)$ is smooth even when the function describing the bottom $y=-H(x / \gamma)$ is discontinuous or non differentiable. The function $M(\xi)$ is time independent and becomes identically one in the case of a constant depth. Moreover, in applications this coefficient is bounded and $\inf _{\mathbb{R}} M(\xi)>0$. These properties will be important to obtain the regularity result and the global existence result of solutions to the Boussinesq system (1.1).

Now, instead of using the terrain-following components of the velocity as in the model derived by Nachbin [12], we express the system (2.9)-(2.10) in terms of the potential velocity $u(\xi, t)$ measured at a fixed intermediate depth, say $\zeta=Z_{0}(\xi)$, with $0<Z_{0}(\xi)<1$ (see [11]) as:

$$
\begin{aligned}
& M(\xi) \eta_{t}+ {\left[\left(1+\frac{\alpha \eta}{M(\xi)}\right) u\right]_{\xi}+\frac{\beta}{2}\left[\left(Z_{0}^{2}-\frac{1}{3}\right) u_{\xi \xi]_{\xi}}=0\right.} \\
& u_{t}+\eta_{\xi}+\alpha\left(\frac{u^{2}}{2 M^{2}(\xi)}\right)_{\xi}+\frac{\beta}{2}\left(Z_{0}^{2}-1\right) u_{\xi \xi t}=0
\end{aligned}
$$

where only the terms up to $O(\alpha), O(\beta)$ have been retained in the equations. In [11] one of the authors studies the impact of the selection of the depth $Z_{0}$ on the dispersive 
characteristics of the model above. In particular, when $Z_{0}^{2}=2 / 3$, system (2.12)-(2.13) takes the form

$$
\begin{array}{r}
M(\xi) \eta_{t}+\left[\left(1+\frac{\alpha \eta}{M(\xi)}\right) u\right]_{\xi}+\frac{\beta}{6} u_{\xi \xi \xi}=0, \\
u_{t}+\eta_{\xi}+\alpha\left(\frac{u^{2}}{2 M^{2}(\xi)}\right)_{\xi}-\frac{\beta}{6} u_{\xi \xi t}=0 .
\end{array}
$$

Appropriate initial conditions for the system above are:

$$
\eta(\xi, 0)=f(\xi), \quad u(\xi, 0)=g(\xi), \quad \xi \in \mathbb{R} .
$$

Formally, the equation (2.14) implies in

$$
u_{\xi}(\xi, t)=-M(\xi) \eta_{t}+O(\alpha, \beta) .
$$

Putting this relationship into system (2.14)-(2.15), and retaining only the terms of order $O(\alpha, \beta)$, we obtain the Boussinesq system (1.1).

Additional Boussinesq formulations are also derived by Muñoz and Nachbin in [11] by changing the velocity variable (i.e. the depth $Z_{0}$ ) in the model (2.12)-(2.13). However, the theoretical advantage of system (1.1) comes from the presence of symmetric dispersive terms of the form $\xi \xi t$, in both the mass and momentum conservation equations. This fact will be important in the next sections in order to prove existence and uniqueness of the Cauchy problem (1.1).

3. Numerical properties of the proposed Boussinesq system. In first place, the linear dispersion relation between $k$ and $\omega$ for system (1.1) is

$$
\omega^{2}=\frac{k^{2}}{\left(1+\frac{\beta}{6} k^{2}\right)^{2}},
$$

which implies that the phase velocity $\omega / k$ of the Fourier components of the wave remains bounded for all wave number $k$. This issue will be crucial to consider the stability of a numerical solver for the Boussinesq system (1.1). Further, we think this system is appropriate for performing numerical simulations for system (1.1) even though the topography is highly oscillatory, in which case the corresponding metric coefficient $M(\xi)$ and $M^{\prime}(\xi)$ would be highly variable. In general, the presence of highly oscillatory coefficients in the dispersive terms of a differential equation may introduce small spurious oscillations in the numerical solutions which would accumulate and become appreciable over a long period of time. This is connected with the stiffness phenomenon. As a consequence, when we gradually decrease the length scale of the topography irregularities, we obtain a wider spectrum range for the corresponding system of ordinary differential equations in the Fourier space.

Our opinion is that this problem gets around in formulation (1.1) in a similar way as in the terrain-following Boussinesq system in [12] (see [10]). The key is the curvilinear coordinate system introduced in the full potential theory equations in the derivation of model (1.1) (see Section 2). Although the topography dependent coefficient $M(\xi)$ is not completely removed from the dispersive terms of system (1.1), they result to be expressed in conservative form. Thus we must not to calculate the 
derivatives of the function $M(\xi)$ in a numerical scheme for system (1.1). In fact, rewrite it as

$$
\left\{\begin{array}{c}
\left(I-\frac{\beta}{6} \partial_{\xi}^{2}\right)\left(M(\xi) \eta_{t}\right)=-\partial_{\xi}\left[\left(1+\frac{\alpha \eta}{M(\xi)}\right) u\right],(\xi, t) \in \mathbb{R} \times[0, \infty) \\
\left(I-\frac{\beta}{6} \partial_{\xi}^{2}\right)\left(u_{t}\right)=-\eta_{\xi}-\frac{\alpha}{2} \partial_{\xi}\left[\left(\frac{u}{M(\xi)}\right)^{2}\right],(\xi, t) \in \mathbb{R} \times[0, \infty) \\
\eta(\xi, 0)=f(\xi), \quad u(\xi, 0)=g(\xi), \quad \xi \in \mathbb{R} .
\end{array}\right.
$$

Then note that the field $M(\xi) \eta_{t}$ can be computed from the first equation in system (3.2) by inverting the linear operator (with constant coefficients) $I-\frac{\beta}{6} \partial_{\xi}^{2}$. To compute the field $u_{t}$, we must invert the same linear operator. For instance, this step can be accomplished by solving two tridiagonal linear systems with the same topography independent matrix forced by the nonlinear terms on the right side of equations (8.1). Observe that we do not need to calculate the derivatives of the coefficient $M(\xi)$ in any part of the process described because the nonlinear terms are written in conservative form. In [10] one of the authors shows that these facts are crucial in order to remove the stiffness phenomenon in the case of the Boussinesq system in [12].

Furthermore, if we invert exactly the linear operator $I-\frac{\beta}{6} \partial_{\xi}^{2}$ and use an acceleration procedure in an analogous way as in [3], we may reduce the computational cost at solving only one tridiagonal linear system whose matrix is topography independent and constant in each time step. The computational cost per time step is of order $G$, where $G$ is the number of grid points used in the spatial discretization. This is comparable to the cost of solving numerically the system in [12] by using the method described in [10].

On the other side, we remark that the symmetric dispersive terms in the equations in system (8.1) make possible to develop unconditionally stable numerical schemes, at least when the channel is of arbitrary finite length, as in [3] where system (1.1) is solved for $M(\xi) \equiv 1$ (flat bottom). This is not straightforward for the terrain-following system in [12] and therefore it represents a significant advantage (in computational cost) of system (1.1) in large time simulations of waves over a coast of disordered depth.

Another advantage of the proposed Boussinesq formulation arises in laboratory experiments, where the fluid velocity $u$ at a finite depth $\left(Z_{0}=\sqrt{2 / 3}\right)$ in model (1.1) can be measured easier than the transversal weighted average of the terrain-following component of the fluid velocity in the Boussinesq model in [12].

4. Some Preliminaries. The main spaces used in the discussion of the local existence, uniqueness and global existence are the Hilbert space $L^{2}(\mathbb{R})=L^{2}$ and the Banach space $\mathcal{L}_{T}^{2}$ for $T>0$, where the later space is defined as the set of functions $f: \mathbb{R} \times[0, T] \rightarrow \mathbb{R}$ such that $f(\cdot, t) \in L^{2}$ for $t \in[0, T]$ and the map $t \rightarrow f(\cdot, t)$ is continuous from $[0, T] \rightarrow L^{2}$. The norm in $\mathcal{L}_{T}^{2}$ is given by

$$
\|u\|_{\mathcal{L}_{T}^{2}}=\sup _{t \in[0, T]}\|u(\cdot, t)\|
$$

where $\|\cdot\|$ means $\|\cdot\|_{L^{2}}$. Now, we say that $u \in \mathcal{L}^{\infty}$, if $u \in \mathcal{L}_{T}^{2}$ for any $T>0$.

To discuss regularity of the solution, we consider the Banach space

$$
C_{b}^{s}=\left\{g: \mathbb{R} \rightarrow \mathbb{R}: \partial_{\xi}^{r} g \text { is bounded for each } 0 \leq r \leq s\right\}
$$


with norm given by

$$
\|g\|_{C_{b}^{s}}=\sum_{r=0}^{s} \sup _{\xi \in \mathbb{R}}\left|\partial_{\xi}^{r} g(\xi)\right|,
$$

and the Banach space $C_{T}^{s}$ defined as the set of functions $u: \mathbb{R} \times[0, T] \rightarrow \mathbb{R}$ such that $u(\cdot, t) \in C_{b}^{s}$ for $t \in[0, T]$ and the map $t \rightarrow u(\cdot, t)$ is continuous from $[0, T] \rightarrow C_{b}^{s}$. The norm in $C_{T}^{s}$ is given by

$$
\|u\|_{C_{T}^{s}}=\sup _{t \in[0, T]}\|u(\cdot, t)\|_{C_{b}^{s}} .
$$

We also requiere to define the space $C_{T}^{s, r}$ as the set of functions $u: \mathbb{R} \times[0, T] \rightarrow \mathbb{R}$ such that $\partial_{t}^{j} u \in C_{T}^{s}$ for $0 \leq j \leq r$. The norm in $C_{T}^{s, r}$ is given by

$$
\|u\|_{C_{T}^{s, r}}=\sum_{j=0}^{r}\left\|\partial_{t}^{j} u\right\|_{C_{T}^{s}} .
$$

We say that $u \in C_{T}^{s, \infty}$, if $u \in C_{T}^{s, r}$ for any $r \in \mathbb{N}$. For $s=0$, we denote $C_{b}^{0}:=C_{b}$ and $C_{T}^{0}:=C_{T}$.

The global existence and uniqueness result for the Cauchy problem (1.1) follows from the local existence and uniqueness result and the existence of a conserved quantity, which allows us to extend in time local solutions.

As mentioned above, the Boussinesq system in (1.1) can be rewritten as the following coupled system of equations

$$
\left\{\begin{aligned}
\left(I-\frac{\beta}{6} \partial_{\xi}^{2}\right) M(\xi) \eta_{t} & =-\partial_{\xi}\left[\left(1+\frac{\alpha \eta}{M(\xi)}\right) u\right] \text { in } \mathbb{R} \times[0, \infty) \\
\left(I-\frac{\beta}{6} \partial_{\xi}^{2}\right) u_{t} & =-\partial_{\xi}\left[\eta+\frac{\alpha}{2}\left(\frac{u}{M(\xi)}\right)^{2}\right] \text { in } \mathbb{R} \times[0, \infty)
\end{aligned}\right.
$$

Now, if we invert the operator $I-\frac{\beta}{6} \partial_{\xi}^{2}$ and integrate over $[0, t]$, we conclude that the pair $(\eta, u)$ satisfies the following system of integral equations,

$$
\begin{aligned}
& \eta(\xi, t)=f(\xi)+\frac{1}{M(\xi)} \int_{0}^{t}\left[K_{\beta} *\left(1+\frac{\alpha \eta}{M(\xi)}\right) u\right](\xi, s) d s, \\
& u(\xi, t)=g(\xi)+\int_{0}^{t}\left[K_{\beta} *\left(\eta+\frac{\alpha u^{2}}{2 M^{2}(\xi)}\right)\right](\xi, s) d s,
\end{aligned}
$$

where $*$ denotes convolution over $\mathbb{R}$ and

$$
K_{\beta}(s)=\frac{3}{\beta} \operatorname{sign}(s) e^{-\sqrt{\frac{6}{\beta}}|s|} .
$$

We observe that the kernel $K_{\beta}$ is a solution in the sense of distributions of the differential equations

$$
K^{\prime}+\sqrt{\frac{6}{\beta}}|K|=\left(\frac{6}{\beta}\right) \delta_{0} \text { and } \quad K^{\prime \prime}-\left(\frac{6}{\beta}\right) K=\left(\frac{6}{\beta}\right) \delta_{0}^{\prime} .
$$


In particular,

$$
\widehat{K_{\beta}}(r)=\frac{-6 r}{6+\beta r^{2}},
$$

where the hat denotes the Fourier transform of $K_{\beta}$

$$
\widehat{K_{\beta}}(r)=\int_{-\infty}^{\infty} K_{\beta}(\xi) e^{-i r \xi} d \xi
$$

Using this fact, we obtain a crucial result related with the convolution between $L^{2}$ functions and the kernel $K_{\beta}$.

LEMma 4.1. Let $K_{\beta}$ be defined as above. Then we have the following properties:

(i) If $v \in L^{2}$, then $K_{\beta} * v$ is bounded, $K_{\beta} * v \in L^{2}$ and we have the estimates

$$
\left\|K_{\beta} * v\right\| \leq c\|v\|, \quad \text { and } \sup _{\mathbb{R}}\left|K_{\beta} * v\right| \leq c\|v\|
$$

(ii) If $v, w \in L^{2}$, then $K_{\beta} *(v w)$ is bounded, $K_{\beta} *(v w) \in L^{2}$ and we have the estimates

$$
\left\|K_{\beta} *(v w)\right\| \leq c\|v\|\|w\|, \quad \text { and } \sup _{\mathbb{R}}\left|K_{\beta} *(v w)\right| \leq c\|v\|\|w\| .
$$

(iii) If $v \in C_{b}, w \in L^{2}$, then $K_{\beta} *(v w)$ is bounded, $K_{\beta} *(v w) \in L^{2}$ and we have the estimates

$$
\left\|K_{\beta} *(v w)\right\| \leq c\|v\|_{C_{b}}\|w\|, \quad \text { and } \sup _{\mathbb{R}}\left|K_{\beta} *(v w)\right| \leq C\|v\|_{C_{b}}\|w\| .
$$

(iv) If $v \in C_{b}^{s}$, then $K_{\beta} * v \in C_{b}^{s+1}$ and

$$
\left\|K_{\beta} * v\right\|_{C_{b}^{s+1}} \leq c\|v\|_{C_{b}^{s}} .
$$

Proof. (i) Let $v \in L^{2}$. By the Cauchy-Schwartz inequality and Plancharel's theorem,

$$
\left|\left(K_{\beta} * v\right)(\xi)\right| \leq \int_{\mathbb{R}}\left|K_{\beta}(\xi-s)\|v(s) \mid d s \leq\| \widehat{K_{\beta}}\|\| v \| .\right.
$$

On the other hand, by Plancharel's theorem

$$
\left\|K_{\beta} * v\right\|^{2}=\left\|\widehat{K_{\beta}} \hat{v}\right\|^{2} \leq \sup _{\mathbb{R}}\left[\frac{6 r}{6+\beta r^{2}}\right]^{2}\|v\|^{2} .
$$

(ii) Let $v, w \in L^{2}$. By the Cauchy-Schwartz inequality,

$$
\left|\left(K_{\beta} *(v w)\right)(\xi)\right| \leq \int_{\mathbb{R}}\left|K_{\beta}(\xi-s)\|v(s)\| w(s)\right| d s \leq \sup _{\mathbb{R}}\left|K_{\beta}\right|\|v\|\|w\| .
$$

On the other hand, for any function $g \in L^{1}(\mathbb{R})$ we have the following elementary property:

$$
\left\|K_{\beta} * g\right\| \leq\left\|K_{\beta}\right\|\|g\|_{L^{1}(\mathbb{R})}
$$


The remaining inequality follows since $v w \in L^{1}(\mathbb{R})$.

(iii) Let $v \in C_{b}$ and $w \in L^{2}$. Since $v w \in L^{2}$, the (i) implies that

$$
\left|\left[K_{\beta} *(v w)\right](\xi)\right| \leq C\|v w\| \leq C\|v\|_{C_{b}}\|w\| .
$$

(iv) Let $v \in C_{b}^{s}$ and let $\Phi=K_{\beta} * v$. By using (4.3), we conclude that

$$
\Phi^{\prime}=-\sqrt{\frac{6}{\beta}}\left(\left|K_{\beta}\right| * v\right)+\left(\frac{6}{\beta}\right) v \quad \text { and } \Phi^{(k+2)}=\left(\frac{6}{\beta}\right) \Phi^{(k)}+\left(\frac{6}{\beta}\right) v^{(k+1)}, k \geq 0 .
$$

To get the estimates, one has to observe that

$$
\|f * g\|_{C_{b}} \leq\|f\|_{C_{b}}\|g\|_{L^{1}}
$$

口

5. Uniqueness. The goal of this section is to prove the uniqueness of the solutions to the Cauchy problem (1.1). The main tools are the integral formulation (4.2), estimatives in Lemma 4.1 for the kernel $K_{\beta}(r)$ and Gronwall's inequality.

THEOREM 5.1. Given $f, g \in L^{2}$, there exists at most one solution pair $(\eta, u)$ defined on $\mathbb{R} \times[0, T]$ to the integral system (4.2) such that $(\eta, u) \in \mathcal{L}_{T}^{2} \times \mathcal{L}_{T}^{2}$.

Proof. Let $\left(\eta_{i}, u_{i}\right)(\mathrm{i}=1,2)$ be two solutions of the integral system (4.2). Let $\eta=\eta_{1}-\eta_{2}$ and $u=u_{1}-u_{2}$. Then the pair $(\eta, u)$ satisfies the couple system of integral equations

$$
\begin{aligned}
& \eta(\xi, t)=\frac{1}{M(\xi)} \int_{0}^{t}\left[K_{\beta} *\left(\left(1+\frac{\alpha \eta_{2}}{M(\xi)}\right) u+\left(\frac{\alpha}{M(\xi)}\right) \eta u_{1}\right)\right](\xi, s) d s, \\
& u(\xi, t)=\int_{0}^{t}\left[K_{\beta} *\left(\eta+\left(\frac{\alpha}{2 M^{2}(\xi)}\right)\left(u_{1}+u_{2}\right) u\right)\right](\xi, s) d s .
\end{aligned}
$$

In consequence, we have that

$$
\begin{aligned}
& \|\eta(\cdot, t)\| \leq C\left(M, K_{\beta}, \alpha\right) \int_{0}^{t}\left[\|u(\cdot, s)\|+\left\|\eta_{2}(\cdot, s)\right\|\|u(\cdot, s)\|+\|\eta(\cdot, s)\|\left\|u_{1}(\cdot, s)\right\|\right] d s, \\
& \|u(\cdot, t)\| \leq C\left(M, K_{\beta}, \alpha\right) \int_{0}^{t}\left[\|\eta(\cdot, s)\|+\left(\left\|u_{1}(\cdot, s)\right\|+\left\|u_{2}(\cdot, s)\right\|\right)\|u(\cdot, s)\|\right] d s
\end{aligned}
$$

Using previous inequalities and the hypotheses on $\eta_{i}$ and $u_{i}$ we conclude that the pair $(\eta(\cdot, t), u(\cdot, t))$ satisfies for $t \in[0, T]$,

$$
\|\eta(\cdot, t)\|+\|u(\cdot, t)\| \leq D \int_{0}^{t}(\|\eta(\cdot, s)\|+\|u(\cdot, s)\|) d s,
$$

where $D \leq C\left(M, K_{\beta}, \alpha\right)\left(1+\left\|\eta_{2}\right\|_{\mathcal{L}_{T}^{2}}+\left\|u_{1}\right\|_{\mathcal{L}_{T}^{2}}+\left\|u_{2}\right\|_{\mathcal{L}_{T}^{2}}\right)$. Gronwall's inequality implies that $\|\eta(\cdot, t)\|+\|u(\cdot, t)\|=0$ for $t \in[0, T]$. $\mathrm{C}$ 
6. Local Existence. In this section, we demonstrate the existence of solutions to the Cauchy problem associated with the Boussinesq system (1.1) in a small time interval. This is achieved by applying the contraction mapping theorem to the integral operator associated to the formulation (4.2). We consider initial data in $L^{2}$ and in $C_{b}^{s}$.

Theorem 6.1. Let $f$ and $g$ be functions in $L^{2}$ and let $b=\|f\|+\|g\|$. Then, there exists $T=T(b)$ such that the integral system (4.2) has a solution pair $(\eta, u) \in$ $\mathcal{L}_{T}^{2} \times \mathcal{L}_{T}^{2}$.

Proof. Let $E_{T}$ be defined as the Banach space $E_{T}=\mathcal{L}_{T}^{2} \times \mathcal{L}_{T}^{2}$ with product norm and let $\mathcal{A}$ be the integral operator defined as

$$
\mathcal{A}\left(\begin{array}{l}
\eta \\
u
\end{array}\right)=\left(\begin{array}{c}
f(\xi)+\frac{1}{M(\xi)} \int_{0}^{t}\left[K_{\beta} *\left(1+\frac{\alpha \eta}{M(\xi)}\right) u\right](\xi, s) d s \\
g(\xi)+\int_{0}^{t}\left[K_{\beta} *\left(\eta+\frac{\alpha u^{2}}{2 M^{2}(\xi)}\right)\right](\xi, s) d s
\end{array}\right) .
$$

We will see that a solution of the integral system is characterized as a fixed point of the operator $\mathcal{A}$ on $E_{T}$ by using the contraction mapping principle, for $T$ small enough.

The first observation is that as a consequence of Lemma 4.1, $\mathcal{A}$ maps $E_{T}$ into itself. On the other hand, given $\left(\eta_{1}, u_{1}\right)$ and $\left(\eta_{2}, u_{2}\right)$ in $E_{T}$, we conclude that for some positive constant $C=C\left(M, \alpha, K_{\beta}\right)$,

$$
\begin{array}{r}
\left\|\mathcal{A}\left(\begin{array}{l}
\eta_{1} \\
u_{1}
\end{array}\right)-\mathcal{A}\left(\begin{array}{l}
\eta_{2} \\
u_{2}
\end{array}\right)\right\|_{E_{T}} \leq C T\left[2+\left\|\eta_{2}\right\|_{\mathcal{L}_{T}^{2}}+2\left\|u_{1}\right\|_{\mathcal{L}_{T}^{2}}+\left\|u_{2}\right\|_{\mathcal{L}_{T}^{2}}\right] . \\
\left\|\left(\eta_{1}, u_{1}\right)-\left(\eta_{2}, u_{2}\right)\right\|_{E_{T}} .
\end{array}
$$

In fact, using the Hölder inequality and Lemma 4.1, we get the following estimates,

$$
\begin{aligned}
\| \mathcal{A}\left(\begin{array}{l}
\eta_{1} \\
u_{1}
\end{array}\right)- & \mathcal{A}\left(\begin{array}{l}
\eta_{2} \\
u_{2}
\end{array}\right)\left\|_{E_{T}}=\right\| \int_{0}^{t} K_{\beta} *\left(\left(\eta_{1}-\eta_{2}\right)+\frac{\alpha\left(u_{1}-u_{2}\right)\left(u_{1}+u_{2}\right)}{2 M^{2}(\xi)}\right)(\xi, s) d s \|_{\mathcal{L}_{T}^{2}} \\
& +\left\|\int_{0}^{t} \frac{1}{M(\xi)} K_{\beta} *\left(\left(u_{1}-u_{2}\right)\left(1+\frac{\alpha \eta_{2}}{M(\xi)}\right)+\frac{\alpha\left(\eta_{1}-\eta_{2}\right) u_{1}}{M(\xi)}\right)(\xi, s) d s\right\|_{\mathcal{L}_{T}^{2}} \\
\leq & T C\left[\left(1+\left\|\eta_{2}\right\|_{\mathcal{L}_{T}^{2}}\right)\left\|u_{1}-u_{2}\right\|_{\mathcal{L}_{T}^{2}}+\left\|\eta_{1}-\eta_{2}\right\|_{\mathcal{L}_{T}^{2}}\left(1+\left\|u_{1}\right\|_{\mathcal{L}_{T}^{2}}\right)\right. \\
& \left.+\left(\left\|u_{1}\right\|_{\mathcal{L}_{T}^{2}}+\left\|u_{2}\right\|_{\mathcal{L}_{T}^{2}}\right)\left\|u_{1}-u_{2}\right\|_{\mathcal{L}_{T}^{2}}\right] \\
\leq & T C\left[2+\left\|\eta_{2}\right\|_{\mathcal{L}_{T}^{2}}+2\left\|u_{1}\right\|_{\mathcal{L}_{T}^{2}}+\left\|u_{2}\right\|_{\mathcal{L}_{T}^{2}}\right]\left\|\left(\eta_{1}, u_{1}\right)-\left(\eta_{2}, u_{2}\right)\right\|_{E_{T}},
\end{aligned}
$$

where $C=C\left(M, K_{\beta}, \alpha\right)$. Now suppose that $\left(\eta_{i}, u_{i}\right)$ belongs to the closed ball $B_{R}(0)$ of radius $R$ around zero in $E_{T}$, for $i=1,2$, then we have that

$$
\left\|\mathcal{A}\left(\begin{array}{l}
\eta_{1} \\
u_{1}
\end{array}\right)-\mathcal{A}\left(\begin{array}{l}
\eta_{2} \\
u_{2}
\end{array}\right)\right\|_{E_{T}} \leq T C[2+4 R]\left\|\left(\eta_{1}, u_{1}\right)-\left(\eta_{2}, u_{2}\right)\right\|_{E_{T}} .
$$


So, if we define $\Theta=T C[2+4 R]$, then

$$
\left\|\mathcal{A}\left(\begin{array}{l}
\eta_{1} \\
u_{1}
\end{array}\right)-\mathcal{A}\left(\begin{array}{l}
\eta_{2} \\
u_{2}
\end{array}\right)\right\|_{E_{T}} \leq \Theta\left\|\left(\eta_{1}, u_{1}\right)-\left(\eta_{2}, u_{2}\right)\right\|_{E_{T}} .
$$

Now suppose that $(\eta, u) \in B_{R}(0)$. Then, by applying (6.2),

$$
\begin{aligned}
\left\|\mathcal{A}\left(\begin{array}{l}
\eta \\
u
\end{array}\right)\right\|_{E_{T}} & \leq\left\|\mathcal{A}\left(\begin{array}{l}
\eta \\
u
\end{array}\right)-\mathcal{A}\left(\begin{array}{l}
0 \\
0
\end{array}\right)\right\|_{E_{T}}+\left\|\left(\begin{array}{l}
f \\
g
\end{array}\right)\right\|_{L^{2} \times L^{2}} \\
& \leq \Theta\|(\eta, u)\|_{E_{T}}+\|f\|+\|g\| \\
& \leq \Theta R+b .
\end{aligned}
$$

In consequence, if we have that $\Theta R+b \leq R$, then $\mathcal{A}$ maps $B_{R}(0)$ into $B_{R}(0)$. To reach previous condition, let $\Theta=\frac{1}{2}, T(b)=\frac{1}{2}(C[2+4 R])^{-1}$ and $R=2 b$. Then we have that $\Theta R+b \leq R$ and that $\mathcal{A}$ is a contraction on $B_{R}(0)$. Under those conditions, the contraction mapping principle assures the existence a fixed point for the operator $\mathcal{A}$ on $B_{R}(0)$. In other words, there exists a solution $(\eta, u)$ on $E_{T}$ of the integral system (4.2), as desired.

REMARK 6.1. The same type of estimates may be established in the space $C_{T}^{s}$ to obtain a local existence result in this space. More exactly,

Corollary 6.2. Suppose that $f$ and $g$ are functions in $C_{b}^{s}$. If $b=\|f\|_{C_{b}^{s}}+\|g\|_{C_{b}^{s}}$. Then, there exists $T=T(b)$ such that the integral system (4.2) has a solution pair $(\eta, u) \in C_{T}^{s} \times C_{T}^{s}$.

7. Regularity of solutions. This section explores the regularity of the solutions obtained in the Theorem 6.1. Again the regularizing properties of the kernel $K_{\beta}$ (in Lemma 4.1) play an important role.

Theorem 7.1. Let $(\eta, u) \in \mathcal{L}_{T}^{2} \times \mathcal{L}_{T}^{2}$ be the solution of (4.2) on $[0, T]$ corresponding to initial data $(f, g)$. Suppose that $f, g \in L^{2} \cap C_{b}^{2}$ and $M \in C_{b}^{2}$. Then $(\eta, u) \in C_{T}^{2, \infty} \times C_{T}^{2, \infty}$.

Proof. Suppose first that $f, g \in L^{2} \cap C_{b}^{2}$. Now, a straightforward computation using Lemma 4.1 shows that for some constant $C$ depending on $K_{\beta}, \alpha$ and $M$

$$
\begin{aligned}
\|\eta(\cdot, t)\|_{C_{b}}=\sup _{\xi \in \mathbb{R}}|\eta(\xi, t)| & \leq\|f\|_{C_{b}}+C \int_{0}^{t}(1+\|\eta(\cdot, s)\|)\|u(\cdot, s)\| d s \\
& \leq\|f\|_{C_{b}}+C T\left(1+\|\eta\|_{\mathcal{L}_{T}^{2}}\right)\|u\|_{\mathcal{L}_{T}^{2}}, \\
\|u(., t)\|_{C_{b}}=\sup _{\xi \in \mathbb{R}}|u(\xi, t)| & \leq\|g\|_{C_{b}}+C \int_{0}^{t}\left(\|\eta(\cdot, s)\|+\|u(\cdot, s)\|^{2}\right) d s \\
& \leq\|g\|_{C_{b}}+C T\left(\|\eta\|_{\mathcal{L}_{T}^{2}}+\|u\|_{\mathcal{L}_{T}^{2}}^{2}\right)
\end{aligned}
$$


In other words, $\eta, u \in C_{T}^{0}$. On the other hand, by equation (4.3) we have that

$$
\begin{gathered}
(M \eta)_{\xi}=(M f)^{\prime}+\int_{0}^{t}\left\{\left(\frac{6}{\beta}\right)\left(1+\frac{\alpha \eta}{M(\xi)}\right)-\sqrt{\left(\frac{6}{\beta}\right)}\left[\left|K_{\beta}\right| *\left(1+\frac{\alpha \eta}{M(\xi)}\right) u\right] d s,\right. \\
(u)_{\xi}=g^{\prime}+\int_{0}^{t}\left\{\left(\frac{6}{\beta}\right)\left(\eta+\frac{\alpha u^{2}}{2 M^{2}(\xi)}\right)-\sqrt{\left(\frac{6}{\beta}\right)}\left[\left|K_{\beta}\right| *\left(\eta+\frac{\alpha u^{2}}{2 M^{2}(\xi)}\right)\right]\right\} d s .
\end{gathered}
$$

Note that

$$
|| K_{\beta}\left|*\left(1+\frac{\alpha \eta}{M(\xi)}\right) u\right| \leq\left\|K_{\beta}\right\|\left\|\left(1+\frac{\alpha \eta}{M(\xi)}\right) u\right\| \leq C\left(1+\|\eta\|_{C_{T}}\right)\|u\|_{\mathcal{L}_{T}^{2}},
$$

and

$$
|| K_{\beta}\left|*\left(\eta+\frac{\alpha u^{2}}{2 M^{2}(\xi)}\right)\right| \leq\left\|K_{\beta}\right\|\left\|\eta+\frac{\alpha u^{2}}{2 M^{2}(\xi)}\right\| \leq C\left(\|\eta\|_{\mathcal{L}_{T}^{2}}+\|u\|_{C_{T}}\|u\|_{\mathcal{L}_{T}^{2}}\right) .
$$

In consequence $M \eta, u \in C_{T}^{1}$. Using this fact and the recursive formulae for the second derivatives

$$
\begin{gathered}
(M \eta)_{\xi \xi}=(M f)^{\prime \prime}+\left(\frac{6}{\beta}\right) M(\eta-f)+\left(\frac{6}{\beta}\right) \int_{0}^{t}\left[\left(1+\frac{\alpha \eta}{M(\xi)}\right) u\right]_{\xi} d s, \\
(u)_{\xi \xi}=g^{\prime \prime}+\left(\frac{6}{\beta}\right)(u-g)+\left(\frac{6}{\beta}\right) \int_{0}^{t}\left[\eta+\frac{\alpha u^{2}}{2 M^{2}(\xi)}\right]_{\xi} d s,
\end{gathered}
$$

we also conclude that $M \eta, u \in C_{T}^{2}$. Under the assumptions on $M$, we obtain that $\eta$ is already in $C_{T}^{2}$, as desired.

Finally, to establish that $\eta, u \in C_{T}^{2, \infty}$, we have to show that $\partial_{t}^{j} \eta, \partial_{t}^{j} u \in C_{T}^{2}$ for any $j \in \mathbb{N}$. Now, by the fundamental theorem of calculus we have that

$$
\begin{aligned}
& \eta_{t}=\frac{1}{M}\left[K_{\beta} *\left(1+\frac{\alpha \eta}{M(\xi)}\right) u\right] \\
& u_{t}=K_{\beta} *\left(\eta+\frac{\alpha u^{2}}{2 M^{2}(\xi)}\right)
\end{aligned}
$$

Since $\left(1+\frac{\alpha \eta}{M(\xi)}\right) u$ and $\eta+\frac{\alpha u^{2}}{2 M^{2}(\xi)}$ belong to $C_{T}^{2}$, we conclude that $\eta_{t}$ and $u_{t}$ belong to $C_{T}^{2}$ as well (by lemma 4.1). Now, one may show inductively that $\partial_{t}^{m} \eta$ and $\partial_{t}^{m} u$ belong to $C_{T}^{2}$, for each $m \in \mathbb{N}$. Then, $\eta, u \in C_{T}^{2, \infty}$.

We want to point out that by using an induction argument on $s$, it is straightforward to prove that

Corollary 7.2. Let $(\eta, u) \in \mathcal{L}_{T}^{2} \times \mathcal{L}_{T}^{2}$ be the solution of (4.2) on $[0, T]$ corresponding to initial data $(f, g)$. Suppose that $f, g \in L^{2} \cap C_{b}^{s}$ and $M \in C_{b}^{s}$ for some $s \geq 0$. Then $(\eta, u) \in C_{T}^{s, \infty} \times C_{T}^{s, \infty}$. 
The key fact to establish this result is that the $(k+2)$ derivative of $M \eta$ and $u$ are given by,

$$
\begin{aligned}
(M \eta)^{(k+2)} & =(M f)^{(k+2)}+\left(\frac{6}{\beta}\right)(\eta-f)^{(k)}+\left(\frac{6}{\beta}\right) \int_{0}^{t}\left[\left(1+\frac{\alpha \eta}{M(\xi)}\right) u\right]^{(k+1)} d s, \\
(u)^{(k+2)} & =g^{(k+2)}+\left(\frac{6}{\beta}\right)(u-g)^{(k)}+\left(\frac{6}{\beta}\right) \int_{0}^{t}\left[\eta+\frac{\alpha u^{2}}{2 M^{2}(\xi)}\right]^{(k+1)} d s .
\end{aligned}
$$

REMARK 7.1. As mentioned above, due to the equation (2.11) the free surface coefficient $M(\xi)$ is a $C^{\infty}$ function. However, we point out that the proof of Theorem 7.1 only requires the second order derivatives of the coefficient $M(\xi)$.

8. Global existence. As a general principle, global existence results in time follows as a consequence of the local existence results and the existence of a conserved quantity in time which is related with the space norm. For this particular problem, it is not straightforward to find a conserved quantity. However, if we have a solution of the Boussinesq system (1.1) $(\eta, u)$ and we perform the substitution $u(\xi, t)=M(\xi) v(\xi, t)$, then the pair $(\eta, v)$ satisfies the following system with $\tilde{g}=\frac{g}{M}$,

$$
\left\{\begin{array}{c}
M(\xi) \eta_{t}+\partial_{\xi}\left[\left(1+\frac{\alpha \eta}{M(\xi)}\right) M v\right]-\frac{\beta}{6} \partial_{\xi}^{2}\left(M(\xi) \eta_{t}\right)=0,(\xi, t) \in \mathbb{R} \times[0, \infty) \\
M(\xi) v_{t}+\eta_{\xi}+\frac{\alpha}{2} \partial_{\xi}\left[v^{2}\right]-\frac{\beta}{6} \partial_{\xi}^{2}\left(M(\xi) v_{t}\right)=0,(\xi, t) \in \mathbb{R} \times[0, \infty) \\
\eta(\xi, 0)=f(\xi), \quad v(\xi, 0)=\tilde{g}(\xi), \quad \xi \in \mathbb{R},
\end{array}\right.
$$

Formulation above has analogous numerical properties as system (1.1) (see Section 3) and therefore it is a good candidate for simulating multiscale phenomena of weakly nonlinear, weakly dispersive waves in a channel of disordered depth.

Now, for solutions $(\eta, v)$ of this system, we have the existence of a non linear functional, which is conserved in time on solutions of the integral system. The energy type functional is given by

$$
\mathcal{E}(t)=\mathcal{E}(t, \eta, v)=\frac{1}{2} \int_{\mathbb{R}}\left[\left(1+\frac{\alpha \eta(\xi, t)}{M(\xi)}\right)[M(\xi) v(\xi, t)]^{2}+M(\xi) \eta^{2}(\xi, t)\right] d \xi
$$

Lemma 8.1. Let $(\eta, v)$ be a solution of (8.1) with $\eta, v \in \mathcal{L}_{T}^{2} \cap C_{T}^{2}$ and let $\mathcal{E}$ be defined as (8.2). Then $\mathcal{E}(t)=\mathcal{E}(0)$, for all $t$ in $[0, T]$. 
Proof. Differentiating with respect to $t$, we have that

$$
\begin{aligned}
\mathcal{E}^{\prime}(t)=\int_{\mathbb{R}}\left[\left(1+\frac{\alpha \eta}{M(\xi)}\right) M^{2}(\xi) v v_{t}+\frac{\alpha}{2} M(\xi) v^{2} \eta_{t}+M(\xi) \eta \eta_{t}\right] d \xi \\
=\int_{\mathbb{R}}\left\{M(\xi) v_{t}\left[\left(1+\frac{\alpha \eta}{M(\xi)}\right) M(\xi) v-\frac{\beta}{6} \partial_{\xi}\left[M(\xi) \eta_{t}\right]\right]\right. \\
\left.\quad+M(\xi) \eta_{t}\left[\eta+\frac{\alpha}{2} v^{2}-\frac{\beta}{6} \partial_{\xi}\left[M(\xi) v_{t}\right]\right]+\frac{\beta}{6} \partial_{\xi}\left[\left(M(\xi) v_{t}\right)\left(M(\xi) \eta_{t}\right)\right]\right\} d \xi \\
=\int_{\mathbb{R}}\left\{\left[\left(1+\frac{\alpha \eta}{M(\xi)}\right) M(\xi) v-\frac{\beta}{6} \partial_{\xi}\left[M(\xi) \eta_{t}\right]\right]\left[\eta+\frac{\alpha}{2} v^{2}-\frac{\beta}{6} \partial_{\xi}\left[M(\xi) v_{t}\right]\right]\right\}_{\xi} d \xi \\
=0 .
\end{aligned}
$$

In other words, $\mathcal{E}(t)=\mathcal{E}(0)$.

Theorem 8.2. Let $f, \tilde{g} \in L^{2} \cap C_{b}^{2}(\mathbb{R})$ and suppose that

$$
\sup _{\mathbb{R}}\left[\frac{\alpha|f(\xi)|}{M(\xi)}\right] \leq \rho<1
$$

Then there exists a unique classical solution $(\eta, v)$ of the initial value problem (8.1) which, with its temporal derivatives of all orders, lies in $\mathcal{L}^{\infty} \times \mathcal{L}^{\infty}$ and in $C_{T}^{2} \times C_{T}^{2}$ for each $T>0$.

Proof. Let $B_{1}$ an upper bound for $\|f\|+\|\tilde{g}\|$, which will be chosen properly below. By the local existence result, there is a solution $(\eta, v)$ in $E_{T}$ for $T=T\left(B_{1}\right)$. Since $\eta \in C_{T}$, the map, $t \rightarrow \eta(\cdot, t)$ from $[0, T]$ to $C_{b}$ is continuous. Then, there exists at least for some small time interval, $\left[0, t_{0}\right]$, in which for $t \in\left[0, t_{0}\right]$,

$$
0<1-\rho \leq 1+\frac{\alpha \eta(\xi, t)}{M(\xi)} \leq 1+\rho
$$

Since $-1<-\rho \leq \inf _{\xi \in \mathbb{R}} \frac{\alpha \eta\left(\xi, t_{0}\right)}{M(\xi)} \leq \frac{\alpha \eta\left(\xi, t_{0}\right)}{M(\xi)} \leq \sup _{\xi \in \mathbb{R}} \frac{\alpha \eta\left(\xi, t_{0}\right)}{M(\xi)} \leq \rho<1$, this argument may be repeated with the general conclusion

$$
\frac{\alpha|\eta(\xi, t)|}{M(\xi)} \leq \rho<1
$$

as long as the solution continue to exist, independent of $t \geq 0$. In fact, let $t \leq t_{0}$. 
Then,

$$
\begin{aligned}
\frac{1}{2}\left(\|v(\cdot, t)\|^{2}+\|\eta(\cdot, t)\|^{2}\right) & =\frac{1}{2} \int_{\mathbb{R}}\left\{v^{2}(\xi, t)+\eta^{2}(\xi, t)\right\} d \xi \\
& \leq \frac{1}{2} \int_{\mathbb{R}}\left\{\frac{1}{(1-\rho) \inf _{\mathbb{R}} M^{2}}\left(1+\frac{\alpha \eta(\xi, t)}{M(\xi)}\right)[M(\xi) v(\xi, t)]^{2}\right. \\
& \left.\quad+\left(\frac{M(\xi)}{\inf _{\mathbb{R}} M}\right) \eta^{2}(\xi, t)\right\} d \xi \\
& \leq \max \left\{\frac{1}{(1-\rho) \inf _{\mathbb{R}} M^{2}}, \frac{1}{\inf _{\mathbb{R}} M}\right\} \mathcal{E}(t) \\
& \leq \max \left\{\frac{1}{(1-\rho) \inf _{\mathbb{R}} M^{2}}, \frac{1}{\inf _{\mathbb{R}} M}\right\} \mathcal{E}(0):=\frac{1}{8} B_{1}^{2} .
\end{aligned}
$$

Now, consider the pair $(\tilde{\eta}, \tilde{v}) \in C_{T} \times C_{T}$, where $\tilde{\eta}(\xi, t)=\eta\left(\xi, t+t_{0}\right)$ and $\tilde{v}(\xi, t)=$ $v\left(\xi, t+t_{0}\right)$. Since $B_{1}$ is now fixed, then previous estimates applied to $(\tilde{\eta}, \tilde{v})$ implies that (8.4) holds for $t_{0} \leq t \leq 2 t_{0}$, and so for $0 \leq t \leq T$, because

$$
\left\|v\left(\cdot, t_{0}\right)\right\|+\left\|\eta\left(\cdot, t_{0}\right)\right\|=\|\tilde{v}(\cdot, 0)\|+\|\tilde{\eta}(\cdot, 0)\| \leq B_{1} .
$$

In consequence, this choosing of $B_{1}$ and that estimate (8.4) holds for $0 \leq t \leq T$ assure that, $\|v(\cdot, t)\|+\|\eta(\cdot, t)\| \leq B_{1}$, independent of $t$. Thus, the contraction mapping principle in Theorem 6.1 may be repeated using $\eta(\cdot, T)$ and $u(\cdot, T)$ as the initial data, to extend $(\eta, u)$ to the interval $\left[0,2 T\left(B_{1}\right)\right]$. Continuing in the way, a global solution to the integral system (4.2) can be defined. Moreover, estimate (8.4) implies that the solution lies in $\mathcal{L}^{\infty} \times \mathcal{L}^{\infty}$. By differentiating successively the integral equation with respect to $t$, one can show that temporal derivatives lie in $\mathcal{L}^{\infty} \times \mathcal{L}^{\infty}$. The regularity claimed follows by Theorem 7.1.

REMARK 8.1. Condition (8.3) is natural from the physical point of view. In first place, $\alpha f(\xi) / M(\xi)>-1$ means that the channel does not run dry at the start. Moreover, the dimensionless parameter $\alpha$ is small for small amplitude waves. Thus, it is reasonable to suppose that $\alpha f(\xi) / M(\xi)<1$.

9. Continuous dependence on initial conditions. We prove the following theorem.

Theorem 9.1. Let $\left(\eta_{1}, v_{1}\right)$ and $\left(\eta_{2}, v_{2}\right)$ in $\left(\mathcal{L}_{T}^{2}, \mathcal{L}_{T}^{2}\right)$, the solution pairs to the system of equations in (8.1), corresponding to the initial data $\left(f_{1}, g_{1}\right)$ and $\left(f_{2}, g_{2}\right)$ which satisfy the hypotheses of Theorem 8.2. Then we have that

$$
\|\eta\|_{\mathcal{L}_{T}^{2}}+\|v\|_{\mathcal{L}_{T}^{2}} \leq(\|f\|+\|g\|) e^{D T}
$$

where $\eta=\eta_{1}-\eta_{2}, v=v_{1}-v_{2}, f=f_{1}-f_{2}$ and $g=g_{1}-g_{2}$. Furthermore, the constant $D$ may be bounded independently of the time.

Proof. Proceeding in a similar way as in the uniqueness proof, we obtain that the pair $(\eta, v)$ satisfies for any $t \in[0, T]$ the system of integral equations

$$
\begin{aligned}
& \eta(\xi, t)=f(\xi)+\frac{1}{M(\xi)} \int_{0}^{t} K_{\beta} *\left[\left(1+\alpha \frac{\eta_{2}}{M}\right) M v+\alpha \eta v_{1}\right](\xi, s) d s \\
& v(\xi, t)=g(\xi)+\frac{1}{M(\xi)} \int_{0}^{t} K_{\beta} *\left(\eta+\frac{\alpha}{2}\left(v_{1}+v_{2}\right) v\right)(\xi, s) d s
\end{aligned}
$$


Therefore by using the estimates of the kernel $K_{\beta}$ in Lemma 4.1 we obtain

$$
\begin{aligned}
& \|\eta(\cdot, t)\| \leq\|f\|+C\left(M, K_{\beta}, \alpha\right) \int_{0}^{t}\left[\|v(\cdot, s)\|+\left\|\eta_{2}(\cdot, s)\right\|\|v(\cdot, s)\|+\|\eta(\cdot, s)\|\left\|v_{1}(\cdot, s)\right\|\right] d s \\
& \|v(\cdot, t)\| \leq\|g\|+C\left(M, K_{\beta}, \alpha\right) \int_{0}^{t}\left[\|\eta(\cdot, s)\|+\left(\left\|v_{1}(\cdot, s)\right\|+\left\|v_{2}(\cdot, s)\right\|\right)\|v(\cdot, s)\|\right] d s .
\end{aligned}
$$

Using previous inequalities and the hypotheses on $\eta_{i}$ and $v_{i}$ we conclude that the pair $(\eta(\cdot, t), u(\cdot, t))$ satisfies for any $t \in[0, T]$,

$$
\|\eta(\cdot, t)\|+\|v(\cdot, t)\| \leq\|f\|+\|g\|+D \int_{0}^{t}(\|\eta(\cdot, s)\|+\|v(\cdot, s)\|) d s
$$

where $D \leq C\left(M, K_{\beta}, \alpha\right)\left(1+\left\|\eta_{2}\right\|_{\mathcal{L}_{T}^{2}}+\left\|v_{1}\right\|_{\mathcal{L}_{T}^{2}}+\left\|v_{2}\right\|_{\mathcal{L}_{T}^{2}}\right) . \quad$ Gronwall's inequality implies the estimate (9.1). Since the initial data satisfy the hypotheses of Theorem 8.2 we also have the estimates

$$
\left\|v_{i}\right\|_{\mathcal{L}_{T}^{2}} \leq \frac{1}{2} B_{1}, \quad\left\|\eta_{i}\right\|_{\mathcal{L}_{T}^{2}} \leq \frac{1}{2} B_{1}, \quad i=1,2
$$

where $B_{1}$ is the (time independent) constant defined in Theorem 8.2 (c.f. equation $(8.5))$.

REMARK 9.1. Note that the constant $B_{1}$ depends only on the dimensionless parameter $\alpha$, the coefficient $M(\xi)$, the initial wave elevation and the initial energy $\mathcal{E}(t=0)$.

10. Conclusions. We established global existence and uniqueness of solutions of a Boussinesq type system forced by highly oscillatory coefficients. In the analysis, we have shown that the solution pair $(\eta, u)$ of the Boussinesq system (1.1) corresponds to a fixed point of the nonlinear operator $\mathcal{A}(\eta, u)$, defined in Section 6 . When the time $T>0$ is small enough, the operator $\mathcal{A}(\eta, u)$ is a contraction in a small enough ball of the space $\mathcal{L}_{T}^{2} \times \mathcal{L}_{T}^{2}$. Thus, the local existence of solutions is guaranteed by the contraction mapping principle. The main ingredient in the uniqueness proof was the Gronwall's inequality. For smooth enough initial data, the local solutions were extended in time by using the nonlinear energy type functional $\mathcal{E}(t)$ (defined in Section 8 ), which is conserved in time along solutions of equations (8.1) obtained through a change of variables in system (1.1). The existence of this quantity is one of the more significant theoretical property of this particular Boussinesq formulation. The global solutions were found to be classical provided the initial data belong to the space $L^{2}(\mathbb{R}) \cap C_{b}^{2}(\mathbb{R})$ and satisfy the condition (8.3). Within the proof of the global existence we found the interesting fact that the wave elevation and the fluid velocity remain bounded independently of time, provided that the initial wave elevation is bounded.

We point out that the technique used in this work can not be extended to other Boussinesq formulations such as the one given by equations (2.12)-(2.13) due to the absence of the regularizing term in $\xi \xi t$ in the mass conservation equation (2.12). In such a case, the space $E_{T}=\mathcal{L}_{T}^{2} \times \mathcal{L}_{T}^{2}$ is not left invariant by the integral operator associated with this Boussinesq system. The existence of global solutions to system (2.12)-(2.13) is an open problem to be considered in a further work. 
System (1.1) is a modification of the terrain-following formulation in [12] with interesting advantages from the numerical point of view. The stiffness problem promoted by the topography is removed by introducing an appropriate system of curvilinear coordinates in the derivation of system (1.1) from the full potential theory equations. Furthermore, we can adapt the unconditionally stable and fourth order accurate algorithm described in [3] for approximating solutions of system (1.1) when the channel is of finite length. The computational cost per time step is of order $G$ where $G$ is the number of grid points used in the spatial discretization. This is comparable to other existing Boussinesq solvers such as that in [10]. To understand numerical and theoretical aspects for system (1.1) would become important in order to study water waves models which are weakly nonlinear and weakly dispersive in presence of a rough medium.

Acknowledgments. This work was supported by the Universidad del Valle, Cali-Colombia.

\section{REFERENCES}

[1] C.J. Amick, Regularity and Uniqueness of Solutions to the Boussinesq system of Equations, J. Differential Equations, 54 (1984), pp. 231-247.

[2] J. Bona And R. Smith, A model for two-way propagation of water waves in a channel, Math. Proc. Camb. Phil. Soc., 79 (1976), pp. 167-182.

[3] J. Bona And M. Chen, A Boussinesq system for two-way propagation of nonlinear dispersive waves, Physica D, 116 (1998), pp. 191-224.

[4] J. Bona And H. Chen, Solitary waves in nonlinear dispersive systems, Discrete and continuous Dynamical systems, Series B, Vol. 2, No. 3, August (2002), pp. 313-378.

[5] T. Driscoll, http://www.math.udel.edu/driscoll/software.

[6] J.P. Fouque, J. Garnier And A. Nachbin, Time reversal for dispersive waves in random media, accepted at SIAM J. Appl. Math., 2003.

[7] J. HAmilton, Differential equations for long-period gravity waves on a fluid of rapidly vaying depth, J. Fluid Mech., 83 (1977), pp. 289-310.

[8] C.C. MeI, The applied dynamics of ocean surface waves, John Wiley, 1983.

[9] J.C. Muñoz Grajales And A. Nachbin, Dispersive wave attenuation and refocusing due to disordered orographic forcing, to appear in SIAM J. Appl. Math.

[10] J.C. MuÑoz Grajales And A. NachBin, Stiff microscale forcing and solitary wave refocusing, submitted for publication SIAM Multiscale Modeling and Simulation, 2004.

[11] J.C. Muñoz Grajales And A. NAChBin, A new set of Boussinesq equations for variable depth. In preparation. 2003.

[12] A. NachBIn, A terrain-following Boussinesq system, SIAM J. Appl. Math., 63:3 (2003), pp. 905-922.

[13] O. Nwogu, Alternative form of Boussinesq equations for nearshore wave propagation, J. Waterway, Port, Coastal and Ocean Engineering, 119 (1993), pp. 618-638.

[14] D.H. Peregrine, Long waves on a beach, J. Fluid Mech., 27 (1967), pp. 815-827.

[15] M.E. SchonbeK, Existence of Solutions for the Boussinesq System of Equations, J. Differential Equations, 42 (1981), pp. 325-352.

[16] S.V. Rajopadhye, M.E. Schonbek and M. Wiegner, Asymptotic Decay for a Generalized Boussinesq system, J. Dynamics and Differential Equations, 11:4 (1999), pp. 595-623.

[17] G.B. Whitham, Linear and nonlinear waves, John Wiley, (1974). 